\title{
It is so difficult to see your GP because family doctors are now nearly extinct
}

\section{Successive reforms to general practice have steadily eroded GPs' ability to practise the "art" of the family doctor, writes David Zigmond}

\section{David Zigmond retired GP and psychiatrist}

We are now long into the dangerous mire of the covid-19 pandemic, but the debate around access to NHS consultations is still very much alive. In general practice, amid increasing patient demand and ongoing struggles to recruit and retain staff, many patients are understandably anxious about these difficulties. ${ }^{12}$ Responding to these public concerns, a news presenter recently asked: "Why is it so difficult to get an appointment to see your family doctor?" This seemed a straightforward question. Yet it was, in its wording, more pregnant in meaning than the presenter had, I think, consciously intended.

Family doctors are now extinct or all but. To talk of "family doctors" is now like talking about "box offices" or "telephone lines": they have, in reality, been superseded, although the habit of using this language may persist. Almost certainly the presenter meant, more accurately, a current GP or even, more precisely, a primary care service provider. Here is the hidden lesson in this slippage: we have such a shortage of doctors in general practice because they cannot now be family doctors. This is no word playing pedantry but an important distinction that has been overlooked or ignored.

"Family doctor" was once an accurate synonym for "general practitioner"-until about 30 years ago. That was because those erstwhile doctors were indeed both: they were generalist primary physicians who-importantly-worked in communities in such a way that they got to know individuals within the patients' own milieux, their families.

Such family doctors typically and importantly worked in smaller (than now) units that were like an occupational home to stable and identifiable professional communities. These doctors therefore had two professional forms of identification and belonging: among their colleagues and among their patients.

This previous stability, locality, and familiarity generated many subtle benefits: senses of security, anchorage, containment, and comfort that can only come from well worn bonds and a more delicate personal understanding of the people we care for and work with-all these can only grow, organically, from shared experiences. These social and interpersonal dimensions of general practice were respected and refined as the "art" of the family doctor. They were the base of care from which the "science" of facts and procedures-treatments-could be more meaningfully and sensitively given. And it was in this way-this mixture of art and science-that family doctors could best look out for, and look after, the patients whom they got to know increasingly well.

The culture of medicine as a humanity guided by science, and of this humanity being mediated through familiarity, was the bedrock of erstwhile general practice. It was largely responsible for the high morale, popularity, and stability of the profession for many decades. Yet I'd argue that serial reforms have now all but eliminated such previously vitalising family doctoring from general practice.

Successive government led reforms have incrementally removed the personal art of medicine so that all attention and resources can be deployed to its science. Nuanced considerations of a patient's social environment and personal history are now all too often eclipsed by imperatives to treatment. Vocational motivation has been displaced by corporate compliance. Headspace and heart space for individuals are crowded out by the demands of a forest of industrialised algorithms and protocols-all applied to people the doctor often does not know and, probably, never will.

This is the environment in which many GPs must now work: one lacking the human nourishment of a collegial or patient "family." Most often they are working in very large health complexes, where any problems that linger are usually relayed and shuttled between different practitioners, rotas, and teams. Doctors cannot here glean the depth of patient knowledge or the satisfaction that comes with personal continuity of care. For patients there is little comforting harbour, refuge, or resonance of familiarity: "it's always someone different." The steadfast buttressing and witnessing of other peoples' lives vanishes.

This is often what it is like being a contemporary GP now that they cannot be true family doctors: the work has become, more and more, a proceduralised series of tasks to be checked off according to the schedules of remote authorities. Meanwhile, there is less and less time for the lives, stories, families, and predicaments of patients. Where can any practitioner find long term satisfaction in such work? And who would want to do it? Fewer and fewer is the answer. ${ }^{3}$

The workforce is now decimated by depersonalising dissatisfaction. That is a crucial, and the most enduring, reason why it is now so difficult to see your GP. No amount of money, training, and recruitment can solve these predicaments that come, inevitably, from our misdirection. What about the mooted further initiatives of primary care networks and integrated 
care systems? Surely such plans-urging ever more gigantism-will make such problems worse.

If GPs were still allowed to be family doctors, the situation would be very different.

Competing interests: none declared.

Provenance and peer review: not commissioned; not peer reviewed.

1 Rees J. GP appointments: Patients 'compete' when phones open. BBC News. 6 October 2021. https://www.bbc.co.uk/news/uk-wales-58804594

2 Haynes L. RCGP warns GPs 'working to the limit' as Starmer criticises GP access. GPonline. 5 January 2022. https://www.gponline.com/rcgp-warns-gps-working-limit-starmer-criticises-gpaccess/article/1736659

3 Bostock N. More than one in three GPs planned to quit within five years even before COVID-19. GPonline. 10 June 2021. https://www.gponline.com/one-three-gps-planned-quit-within-five-yearseven-covid-19/article/1718769 